\title{
Interface Tracking Simulations of Bubbly Flows in PWR Relevant Geometries
}

\author{
Jun Fang ${ }^{1}$, Michel Rasquin ${ }^{2}$, Igor A. Bolotnov ${ }^{1}$ \\ ${ }^{1}$ Department of Nuclear Engineering, \\ North Carolina State University, \\ Raleigh, NC 27695 \\ jfang3@ncsu.edu; igor_bolotnov@ncsu.edu \\ ${ }^{2}$ Aerospace Engineering Department, \\ University of Colorado, \\ Boulder, CO 80309 \\ michel.rasquin@colorado.edu
}

\begin{abstract}
The advances in high performance computing (HPC) have allowed direct numerical simulation (DNS) approach coupled with interface tracking methods (ITM) to perform high fidelity simulations of turbulent bubbly flows in various complex geometries. In this work, we have chosen the geometry of the pressurized water reactor (PWR) core subchannel to perform a set of interface tracking simulations (ITS) with fully resolved liquid turbulence.
\end{abstract}

The presented research utilizes a massively parallel finite-element based code, PHASTA, for the subchannel geometry simulations of bubbly flow turbulence. The main objective for this research is to demonstrate the ITS capabilities in gaining new insight into bubble/turbulence interactions and assisting the development of improved closure laws for multiphase computational fluid dynamics (M-CFD).

Both single- and two-phase turbulent flows were studied within a single PWR subchannel. The analysis of numerical results includes the mean gas and liquid velocity profiles, void fraction distribution and turbulent kinetic energy profiles. Two sets of flow rates and bubble sizes were used in the simulations. The chosen flow rates corresponded to the Reynolds numbers of 29,079 and 80,775 based on channel hydraulic diameter $\left(D_{h}\right)$ and mean velocity. The finite element unstructured grids utilized for these simulations include 53.8 million and 1.11 billion elements, respectively. This has allowed to fully resolve 
all the turbulence scales and the deformable interfaces of individual bubbles. For the two-phase flow simulations, a $1 \%$ bubble volume fraction was used which resulted in 17 bubbles in the smaller case and 262 bubbles in the larger case. In the larger simulation case the size of the resolved bubbles is $0.65 \mathrm{~mm}$ in diameter, and the bulk mesh cell size is about 30 microns.

Those large-scale simulations provide new level of details previously unavailable and were enabled by the excellent scaling performance of our two-phase flow solver and access to the state-of-the-art supercomputing resources. The presented simulations used up to 256 thousand processing threads on the IBM BG/Q supercomputer "Mira” (Argonne National Laboratory).

KEYWORDS: DNS, interface tracking, level set method, two-phase bubbly flows, PWR subchannel

\section{Introduction}

A reliable prediction of the single- and two-phase flows in pressurized water reactor (PWR) rod bundles is critical for both reactor safety and thermal-hydraulics analyses. As a result, the turbulent flow in the reactor cores has been studied for decades and remains an active topic in the research of nuclear community. A considerable amount of literature has been published on experiments of turbulent flow in rod bundles. These studies usually measured the distributions of axial velocity, turbulence kinetic energy, and Reynolds stress with various aspect ratios (Pitch-to-Diameter ratio, $P / D$ ) and Reynolds numbers. Trupp and Azad (1975) measured the spatial distributions of mean velocity, Reynolds stresses and other important quantities as functions of Reynolds number and tube spacing for fully developed flows. The measurement was made with three different spacing ratios (i.e. $P / D$ are $1.20,1.35$ and 1.50), and the Reynolds number ranges from 12,000 to 84,000 . Heat transfer calculations form an important part in the design of nuclear fuel elements, which can only be carried out if information of the velocity field is available (Carajilescov and Todreas, 1976). In Carajilescov's study, experimental measurements of the distributions of the axial velocity, turbulence kinetic energy, and Reynolds stresses were performed using a laser Doppler anemometer (LDA) in a simulated interior subchannel of a triangular rod array with $P / D$ $=1.123$ and $L / D_{h}=77$. Detailed experimental data are very important for both turbulence modeling and code validation; continued experiments were done by Rehme (1989), Wu and Trupp (1993) and many other researchers. The measurement techniques are also being improved over time, and now researchers can obtain more reliable data-base of higher spatial and temporal resolution with state-of-the-art experimental techniques. For example, Dominguez-Ontiveros and Hassan (2009) have recently done a 
non-intrusive experimental investigation of flow behavior inside a transparent $5 \times 5$ rod bundle with spacer grids using particle image velocimetry (PIV).

Due to the complex and extreme nature of realistic PWR conditions, it is very challenging (if not impossible) to conduct realistic pressure/temperature conditions experiments to study the turbulent flows in reactor fuel rod bundles. Instead, the computational methodologies are typically chosen as a practical approach to predict flow behavior under PWR conditions. For instance, the advanced thermal-hydraulic subchannel code COBRA-TF (Thurgood, 1983) is being used worldwide for best-estimation evaluations of nuclear reactor safety margins. Both subchannel and computational fluid dynamics (CFD) methods are being improved as the nuclear industry advances to Generation III+ and Generation IV reactor technology. In a recent version of COBRA-TF, a revised spacer grids model was implemented to account for the turbulent mixing enhancement due to spacers and the lateral flow patterns created by specific configurations of the spacers' structural elements (Avramova, 2007). Conner et al. (2010) presented the Westinghouse CFD methodology to model single-phase, steady-state conditions in PWR fuel assemblies as well as benchmark testing. In the meantime, the tremendous growth of computer power in recent years has led to a renewed interest in studying turbulent flows using direct numerical simulation (DNS) approach. In DNS of turbulence, the fundamental equations of fluid motion (the Navier-Stokes equations) are solved, without turbulence closure assumptions (unlike classic CFD approach, or even more empirical subchannel analysis), with sufficient temporal and spatial resolution to represent all the scales of turbulence down to Kolmogorov scales (Lee et al., 2013; Trofimova et al., 2009). Because of the prediction capability based on first principles calculations, DNS is believed to be a promising approach to complement experimental data for the model development of turbulent flows. Several attempts have been exercised by applying DNS to fully-developed single-phase turbulent flow analysis for pin bundles (Ninokata et al., 2004; Baglietto et al., 2006), but the Reynolds numbers resolved in their DNS are relatively low (up to $\operatorname{Re}_{h}$ of 24,300).

Besides the single-phase analysis, the study of two-phase turbulence phenomena is also of great importance because boiling flows can occur during normal operation or accident conditions in light water reactor (LWR) cores. Many two-phase flow models have been developed for nuclear fuel rod bundle designs. Considering the dependence of bubble diameter on local flow conditions, Končar et al. (2004) did the multidimensional modeling of vertical upward subcooled boiling flow. The modeling was based on a two-fluid approach, and local two-phase flow parameters (e.g. bubble size) were calculated. The CFD-BWR model developed by Ustinenko et al. (2008) allows the detailed analysis of the two-phase coolant flow and heat transfer phenomena in a BWR fuel bundle. This model was further combined with 
the commercial CFD code STAR-CD to calculate location of vapor generation onset, axial temperature profile, and axial and radial void distributions. In the field of nuclear power research, one of the major technological issues is the departure from nucleate boiling (DNB) condition in the fuel assembly (Anglart and Nylund, 1996). In order to address these science and engineering challenges, the development of more reliable two-phase closure laws is highly desired. It can take advantage of the detailed information provided by the high fidelity interface tracking simulations (ITS) of bubbly flows with DNS of liquid turbulence.

DNS of two-phase flows has been studied previously and provided unprecedented insight into complex flow phenomena. Lu and Tryggvason (2008) studied a turbulent bubbly upflow in a vertical channel using front tracking method, and it was observed that the void fraction profile highly depends on the deformability of the simulated bubbles. The extended study done by Dabiri et al. (2013) has revealed that a regime transition of vertical channel bubbly upflow takes place when Eötvös number is between 2.0 and 3.0. Bolotnov et al. studied the turbulent bubbly flows in flat channels with DNS to investigate the bubble distribution and bubbles' influence on the turbulence field (Bolotnov et al., 2011; Bolotnov, 2013). Fang et al. (2013) have implemented a proportional-integral-derivative (PID) controller within level set interface tracking simulations to evaluate the drag and lift forces a single bubble experiencing in laminar uniform shear flows, based on which Thomas et al. (2015) have introduced higher order terms in the controlling model and studied the interfacial forces of single bubbles in laminar medium, laminar high and turbulent high shear flows. Demonstration cases show that the drag coefficients extracted at low shear with various bubble Reynolds numbers can achieve an excellent agreement with experimentally based correlations proposed by Tomiyama et al. (1998).

In the presented research, both single and two-phase turbulence are simulated within a PWR subchannel for Reynolds numbers $\left(\operatorname{Re}_{h}\right)$ of 29,079 and 80,774 (based on the hydraulic diameter and mean velocity). The turbulent flow of Reynolds number of 29,530 has been previously simulated in a flat channel (Bolotnov, 2013) and will be compared with the case with $\operatorname{Re}_{h}$ of 29,079 to investigate the influence of PWR geometry on the turbulent flow structures. Since the mesh size for DNS grows exponentially as $\operatorname{Re}_{h}$ increases (Trofimova et al., 2009), the Reynolds number of 80,774 is chosen as the effort approaching to the simulations with realistic PWR conditions by considering the state-of-the-art computing resources (e.g. IBM BG/Q "Mira" at Argonne National Laboratory, \#6 in top500 as of June 2016). Some preliminary results from the low Reynolds number case $(29,079)$ have been presented by Fang et al. (2014) from the limited statistical data available at that time, and since then much larger dataset has been collected to help us better understand the bubbly turbulence phenomena in the PWR subchannel. By 
processing the instantaneous data provided by DNS, statistical results obtained include the mean gas and liquid velocity profiles, void fraction distribution and turbulent kinetic energy profiles. The novel aspect of current work is that DNS coupled with interface tracking method has been applied to the analysis of turbulent bubbly flows inside the PWR subchannel at highest Reynolds number to date (comparable with experimental capabilities for those geometries), which will help develop more accurate closure laws and ensure a higher quality prediction of single and two-phase turbulent flows for nuclear reactor designs.

\section{Numerical method}

\subsection{Flow solver}

The flow solver being used in the present work is PHASTA, which is a parallel, hierarchic, higher-order accurate, adaptive, stabilized (finite element) transient analysis flow solver for both incompressible and compressible flows. This approach has been shown to be an effective tool for bridging a broad range of length scales in turbulent (Reynolds averaged Navier-Stokes (RANS), large-eddy simulation (LES), detached eddy simulation (DES), DNS) flows (Jansen, 1999; Whiting and Jansen, 2001). PHASTA was the first unstructured grid LES code (Jansen, 1993) and has been applied to turbulent flows ranging from validation benchmarks (channel flow, decay of isotropic turbulence) to complex flows (airfoils at maximum lift, flow over a cavity, near lip jet engine flows and fin-tube heat exchangers). The PHASTA code uses advanced anisotropic adaptive algorithms (Sahni et al., 2006) and the most advanced LES/DES models (Tejada-Martínez and Jansen, 2006; Spalart et al., 2006). This capability has been extended to two-phase flows where we use the level set method to track the boundary between two immiscible fluids (Nagrath et al., 2006; Bolotnov et al., 2011). The highly scalable performance of PHASTA on massively parallel computers has already been demonstrated (Rasquin et al., 2014) (the code has shown good scaling up to $768 \times 1024$ processors on the IBM Blue Gene/Q Mira system.

In the simulations the fluid is assumed to be isothermal and incompressible. The strong form of the incompressible Navier-Stokes (INS) is given by

$$
\begin{array}{cc}
\text { Continuity: } & \frac{\partial u_{i}}{\partial x_{i}}=0 \\
\text { Momentum: } & \rho \frac{\partial u_{i}}{\partial t}+\rho u_{j} \frac{\partial u_{i}}{\partial x_{j}}=-\frac{\partial p}{\partial x_{i}}+\frac{\partial \tau_{i j}}{\partial x_{j}}+f_{i}
\end{array}
$$


where $u_{i}$ is the velocity in the $x_{i}$-direction, $\rho$ denotes the density of the fluid, $p$ the static pressure and $\tau_{i j}$ the viscous stress tensor. For the incompressible flow of a Newtonian fluid, the viscous stress tensor is related to the fluid viscosity and the strain rate tensor as:

$$
\tau_{i j}=2 \mu S_{i j}=\mu\left(\frac{\partial u_{i}}{\partial x_{j}}+\frac{\partial u_{j}}{\partial x_{i}}\right)
$$

In order to extend PHASTA's capability from single-phase to two-phase flows, the level set interface tracking method (Sussman et al., 1998; Sethian, 1999; Sussman et al., 1999) was implemented. The bubble interface is modeled as the zero-level set of a smooth function, $\varphi$, where $\varphi$ is called the first scalar and is represented as the signed distance from the zero-level set. At $\varphi=0$, the level set defines the interface. The scalar, $\varphi$, is advected with the fluid according to the advection Eq. (4) described in Sussman et al. (1998). The liquid phase is indicated by a positive level set, $\varphi>0$, while the gas phase by a negative level set, $\varphi<0$.

$$
\frac{\partial \varphi}{\partial t}+u_{j} \frac{\partial \varphi}{\partial x_{j}}=0
$$

The Continuum Surface Force (CSF) model (Brackbill et al., 1992) is used in PHASTA to compute the surface tension force as a local interfacial force density, which is included in the body force term $\left(f_{i}\right)$ on the right hand side of the INS equations.

Evaluating the jump in physical properties across the interface using a step change is challenging numerically; therefore, the properties near the interface are defined using a smoothed Heaviside kernel function, $H_{\varepsilon}$ (Sussman et al., 1999). While the solution may be relatively good in the close vicinity of the interface, the distance field, $\varphi$, may not be correct elsewhere in the domain where the varying fluid velocities throughout the flow field distort the level set contours (such as in a fully resolved turbulent flow). To maintain a true distance field, the level set is corrected at every time iteration with a redistancing operation (Fatemi and Sussman, 1995). A detailed description of the equations and redistancing process used can be found in Bolotnov et al. (2011). 


\subsection{DNS mesh design}

The following requirements must be met to ensure an accurate representation of all relevant scales in PHASTA simulations: (a) the computational domain must be sufficiently large to contain the largest turbulent eddies, and (b) the grid spacing must be sufficiently fine in order to capture the smaller scales of interest (e.g. Kolmogorov turbulent length scale). The first requirement is met if two-point correlations in the stream-wise and span-wise directions vanish within one-half of the computational domain (Trofimova et al., 2009). Meanwhile, the number of mesh points in physical domain must be chosen to resolve the finest scale of appreciable excitation, namely layers of the Kolmogorov dissipation scale thickness (Orszag, 1970). In order to meet the second prerequisite, the first plane of grid points off the walls is designed to have an equivalent distance of $\Delta y^{+}=1.0$. The normalized wall distance is discussed by Pope (2000). More discussions regarding the DNS resolution requirements for turbulent flows can also be found in Kim et al. (1987) and Moser et al. (1999).

\section{Problem description}

To create a single PWR subchannel domain, the model is first built in CAD software (e.g. SolidWorks), which can be then utilized by meshing tools (provided by Simmetrix, Inc. in our case) to generate the corresponding unstructured mesh. Certain number of boundary layers is specified near the fuel rod surface to capture the detailed information regarding the turbulence in the region very close to walls, governed by the law of the wall (Pope, 2000). The mesh size is 53.8 million elements for the case with $R e_{h}$ of 29,079 . Recent progress in advanced parallel meshing tool allows us to generate much larger meshes to fully resolve the turbulence of higher Reynolds numbers, and for the case with $R e_{h}$ of 80,774 the mesh created includes 1.11 billion elements partitioned into 131,072 parts. Both the domain overview and a zoom-in view for the boundary layers are shown in Figure 1. The length of the subchannel corresponds to about 3 hydraulic diameters $(40.5 \mathrm{~mm})$, which is calculated based on the cross section area $(A)$ and perimeter $\left(P_{w}\right)$ illustrated by Eq. (5). The direction of gravity is opposite to the mean flow direction, which represents an upward flow in a vertical subchannel. The cases of two Reynolds numbers are labeled with RE01 (for $\mathrm{Re}_{h}$ of 29,079) and RE02 (for $\mathrm{Re}_{h}$ of 80,774). More detailed discretization parameters are listed in Table I, including domain sizes and resolutions.

$$
D_{h}=\frac{4 A}{P_{w}}
$$


Table I. Discretization parameters

\begin{tabular}{|c|c|c|}
\hline Case & RE01 & RE02 \\
\hline Domain sizes $(\mathrm{mm})$ & \multicolumn{2}{|c|}{$40.5 \times 12.6 \times 12.6$} \\
\hline Rod radius (mm) & \multicolumn{2}{|c|}{4.57} \\
\hline Pitch-to-Diameter Ratio $(P / D)$ & \multicolumn{2}{|c|}{1.38} \\
\hline Reynolds number resolved $\left(\operatorname{Re}_{h}\right)$ & 29,079 & 80,774 \\
\hline Resolved bubble diameter (mm) & 1.6210 & 0.6509 \\
\hline Bulk resolution (mm) & $8.11 \times 10^{-2}$ & $3.25 \times 10^{-2}$ \\
\hline Bubble surface CFL number & 0.21 & 0.39 \\
\hline Maximum CFL number in the domain & 7.5 & 8.0 \\
\hline Thickness of first B. L. $\left(\mathrm{y}^{+}=1\right)(\mathrm{mm})$ & $8.11 \times 10^{-3}$ & $3.25 \times 10^{-3}$ \\
\hline Number of boundary Layers & 13 & 13 \\
\hline Number of points & $9,249,506$ & $186,825,949$ \\
\hline Number of elements & $53,837,248$ & $1,111,168,768$ \\
\hline Number of computing cores used & 8,192 & 131,072 \\
\hline Element per core & 6,572 & 8,478 \\
\hline
\end{tabular}
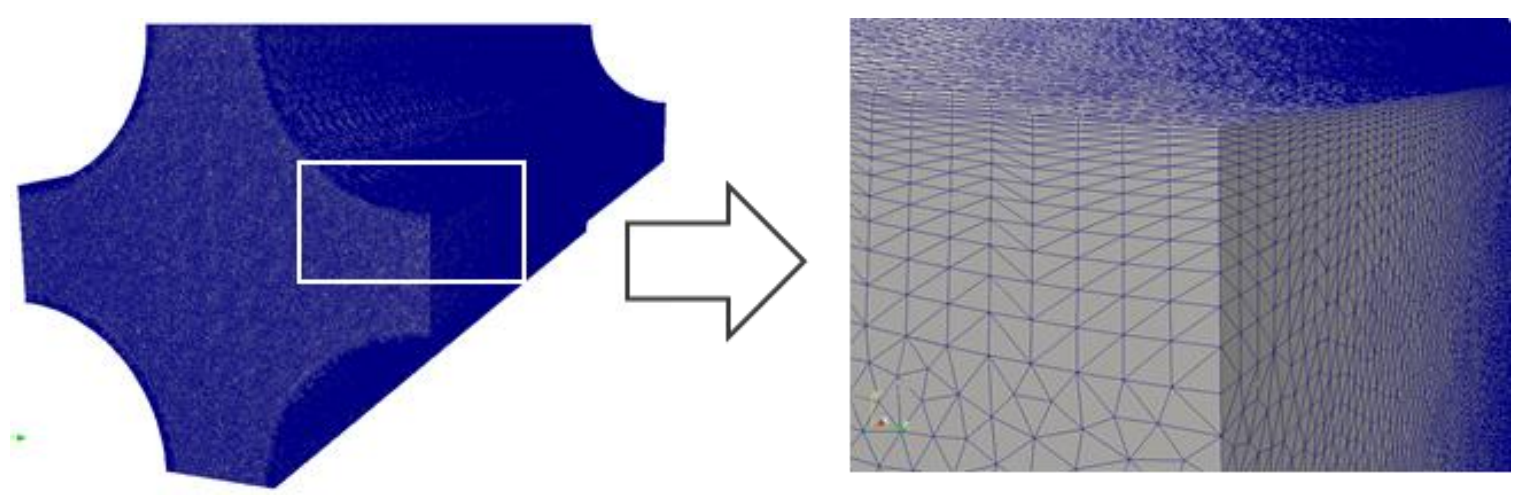

Figure 1. Typical unstructured mesh with boundary layers 
Periodic boundary conditions are utilized to represent a much longer domain than computationally feasible in DNS approach and to be able to achieve statistically steady state flow conditions. The domain is periodic at inflow and outflow planes as well as the transverse faces, and no-slip wall conditions are applied to the fuel rod surface (Figure 2).

The DNS turbulent results for both single- and two-phase flows are produced efficiently using a two-step approach. The single-phase turbulent velocity profile is first generated by placing a sphere blockage region at the domain center to create fluctuations. After large turbulence structures are observed, the barrier is removed and the simulation is carried on till sustained turbulence is established. The statistical data is recorded at this point; the convergent behavior is observed as steady state is achieved as shown in Figure 7. When we ensured that the single-phase turbulence has achieved statistically steady state flow conditions by comparing averaged velocity profiles over different time windows, the second step was performed to initialize the bubbles (representing a $1 \%$ bubble volume fraction). Since gas bubbles are lighter compared to the liquid, the pressure gradient is adjusted to compensate the density change of bulk flow and sustain a constant mean flow velocity. Bubbles' motion and deformation are resolved using level-set interface tracking method. The detailed bubble initialization process has been described previously in Fang et al. (2014).

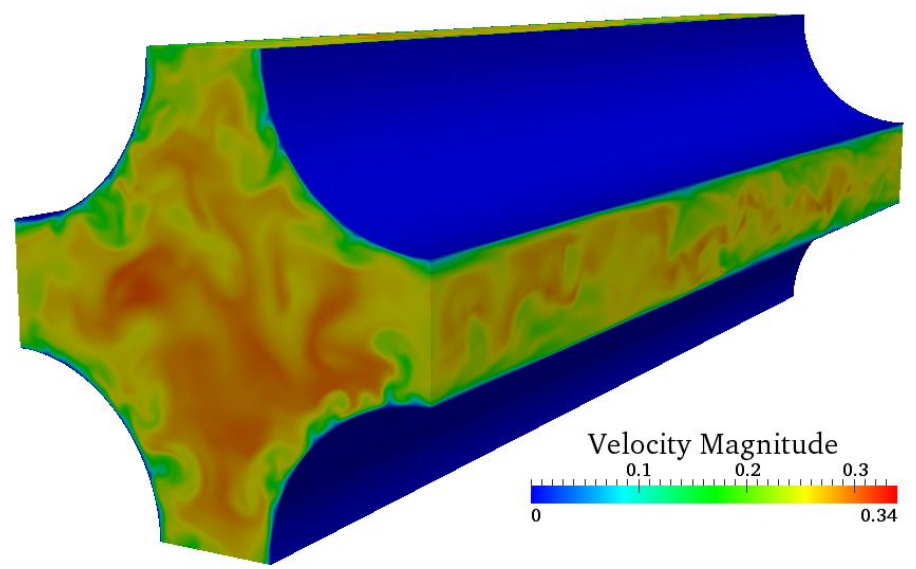

Figure 2. Wall condition in the subchannel simulations

Considering computational cost and quality of the results (based on previous resolution and validation studies), the bubble resolution is set to be 20 elements across diameter, which results in 17 bubbles for the $53.8 \mathrm{M}$ mesh and 262 bubbles for the $1.11 \mathrm{~B}$ mesh at the $1 \%$ volumetric fraction. The scaling studies have shown that the most efficient approach is to use 4 message-passing-interface (MPI) partitions per physical 
core on Mira supercomputer. The computational costs are estimated for both cases, and it takes $68 \mathrm{~K}$ corehours for case RE01 and 1.91M core-hours for case RE02 to achieve one domain flow-through (about $3.14 L / D_{h}$ length) in two-phase simulations. Higher resolution will result in the rapid increase of computational cost while lower resolution is not capable to capture enough details regarding bubbles' behavior to reach meaningful conclusions. As shown in Figure 5, a set of virtual probes are designed and placed in the simulated domain to record instantaneous velocity fluctuations and bubble distribution information. The initial bubble distribution and velocity profile for 17 bubbles and 262 bubbles are shown in Figure 3 and Figure 4 (the direction of mean flow is from left to right as pointed by the red arrow at bottom-left of figures). Interface tracking simulations are run with the bubbles to allow the flow to further develop and the bubbles to achieve their terminal velocities.
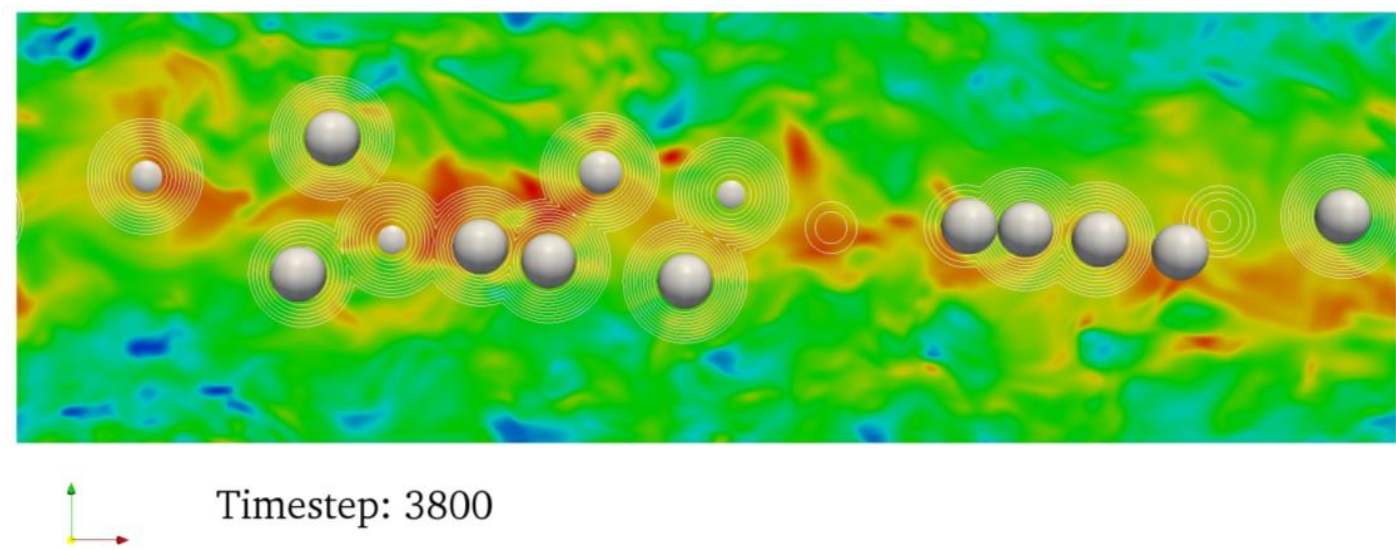

Figure 3. Distribution of 17 bubbles in the turbulent flow (half of the domain can be seen).

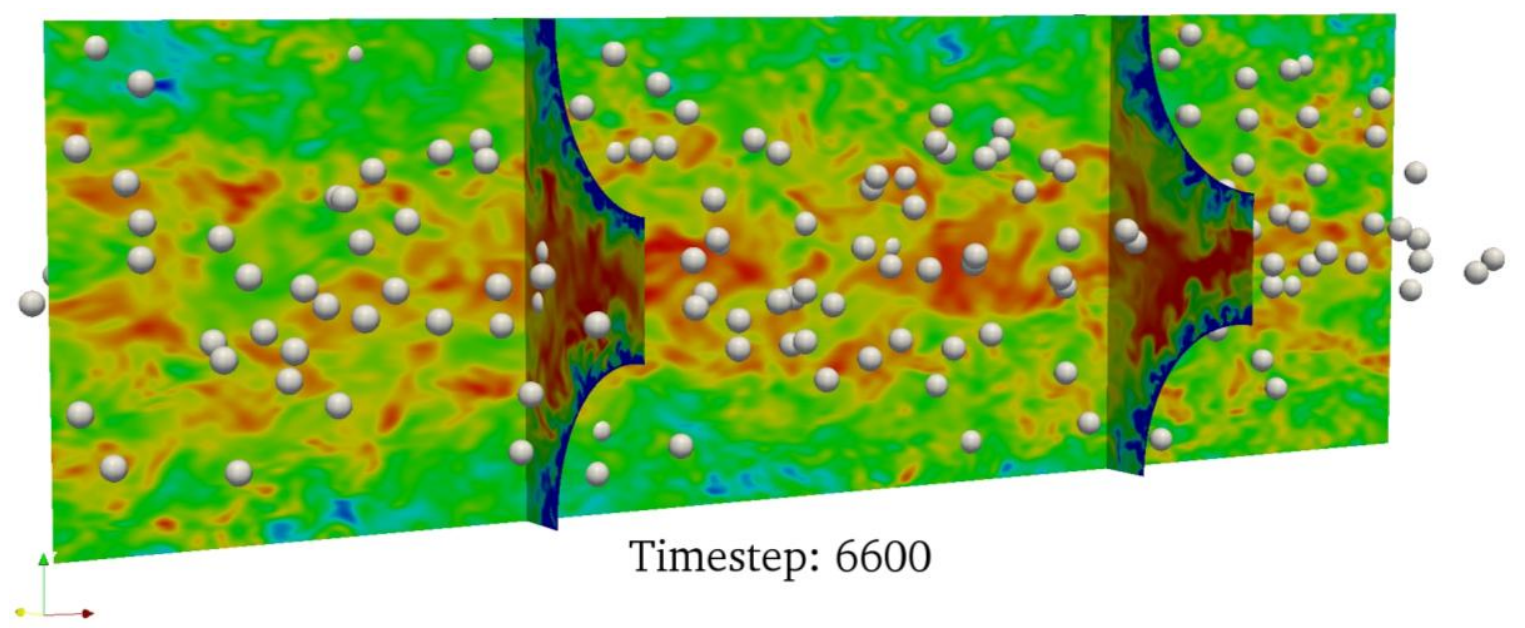

Figure 4. Distribution of 262 bubbles in the turbulent flow 
The key computational parameters and fluid properties are listed in Table II. The viscosities and densities of liquid/gas are determined by using the saturated properties of water and vapor at $300{ }^{\circ} \mathrm{C}$. The realistic PWR conditions were estimated based on the technical parameters provided in AP1000 Design Control Document (Winters et al., 2004). The data collected from the simulations is processed to obtain, for instance, the mean velocity $\left(U_{i}\right)$ and turbulent kinetic energy $(k)$ that are calculated based on Eqs. (6) and (7). The probes used to extract the flow statistics are shown in Figure 5 and their location has been improved based on the previous design used in Fang et al. (2014).

$$
\begin{gathered}
U_{i}(t)=\frac{1}{N_{w}} \sum_{j=1}^{N_{w}} u_{i}\left(t+t_{j}\right) \\
k(t)=\frac{1}{N_{w}} \sum_{j=1}^{N_{w}} \sum_{i=1}^{3} \frac{1}{2} u_{i}^{\prime}\left(t+t_{j}\right)^{2}
\end{gathered}
$$

where, $u_{i}^{\prime}\left(t+t_{j}\right)=u_{i}\left(t+t_{j}\right)-U_{i}(t)$ is the fluctuation of velocity component- $i$ computed at the time instant $t+t_{j} ; N_{w}$ is the number of velocity samples in each time window, $t$ is the current time, $t_{j}=(j-$ $\left.N_{w} / 2\right) \Delta t$ is the local window time, and $\Delta t$ is the time step. For two-phase flows additional parameters, such as void fraction and phasic velocities can also be determined using this statistical analysis method.

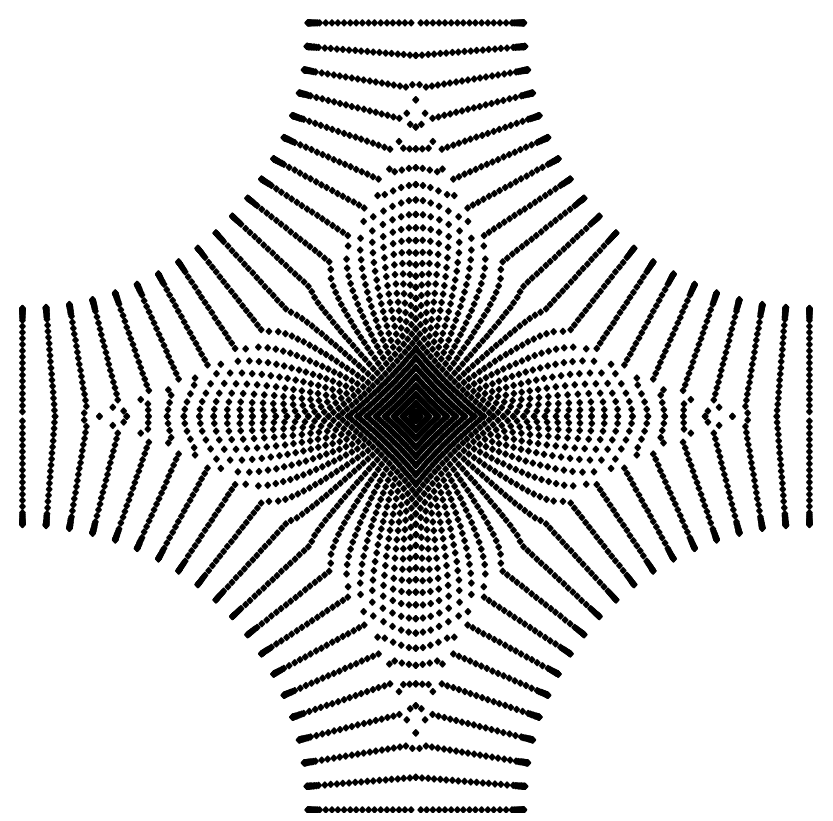

Figure 5. Virtual probe locations used to capture the flow statistics in a single subchannel. 
Table II. Fluid properties used in the simulations

\begin{tabular}{|c|c|c|c|}
\hline Case & RE01 & RE02 & Realistic PWR condition \\
\hline Liquid/Gas Viscosities $(\mathrm{Pa} \cdot \mathrm{s})$ & \multicolumn{3}{|c|}{$8.585 \times 10^{-5} ; 1.965 \times 10^{-5}$} \\
\hline Liquid/Gas Densities $\left(\mathrm{kg} / \mathrm{m}^{3}\right)$ & \multicolumn{3}{|c|}{$712.22 ; 46.17$} \\
\hline Mean velocity $(\mathrm{m} / \mathrm{s})$ & 0.27 & 0.75 & 4.62 \\
\hline Reynolds number $\left(\operatorname{Re}_{h}\right)$ & 29,079 & 80,774 & 452,500 \\
\hline
\end{tabular}

\section{Results and discussion}

Both single- and two-phase subchannel simulations were performed on IBM BG/Q "Mira" at the Leadership Computing Facility (ALCF) located at the Argonne National Laboratory. The simulation results were visualized using the open-source software, ParaView. The scaling performance of the parallel DNS flow solver, PHASTA, was investigated for subchannel cases before the production runs. A set of standard parameters were adopted in all tests and the only difference is the number of mesh partitions. The results obtained confirmed our previous experience (Rasquin et al., 2014) that PHASTA scales very well for massively parallel computations of interest. The simulation efficiency is measured in core-hours consumed to conduct one full time step for each 1 billion elements. Each node on "Mira" has 16 cores while each core is able to perform up to 4 MPI processes, and we observed that 4-mpi per core runs lead to the most efficient simulations, which means 4-mpi per core runs consume the least core-hours with the same mesh partitions (Figure 6). The computational efficiency in cases with smaller partition counts is not as good because the number of elements each core has to compute exceeds the limit that a core can efficiently handle due to memory cache limits. As expected, for partitions numbers higher than 65,536, the efficiency is slightly reduced due to increasing burden from inter-processor communications. The scaling results obtained with the PHASTA code demonstrate that this is one of a very few massively parallel flow solvers capable of full utilization of the largest supercomputers. 


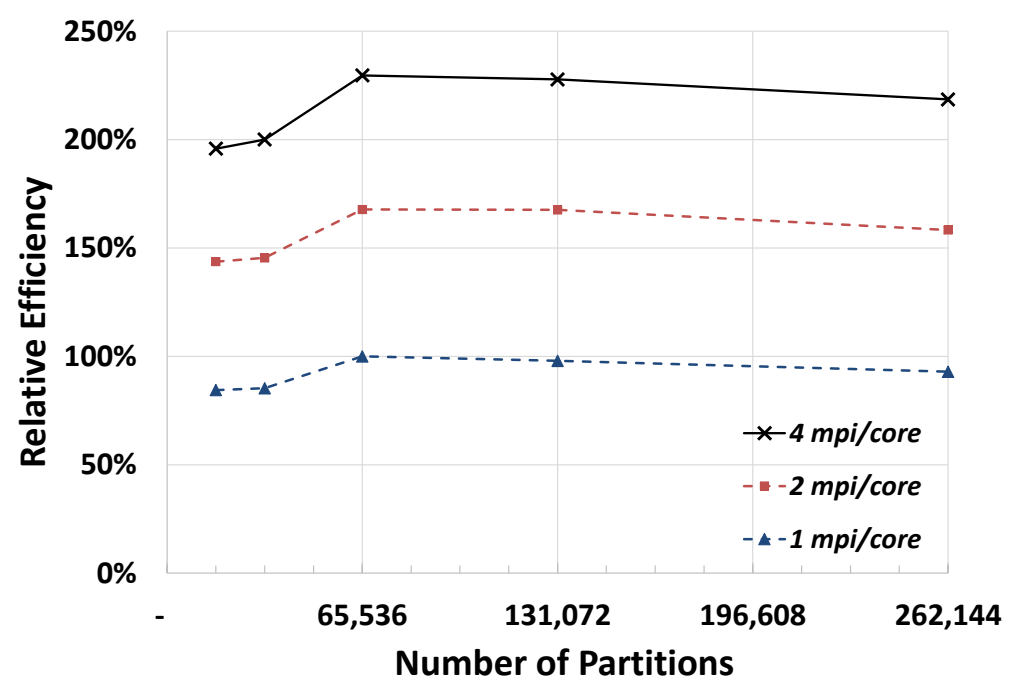

Figure 6. Scaling results of PHASTA on Mira BG/Q with a 1.11 B element mesh (subchannel geometry) normalized using $1 \mathrm{mpi} /$ core run at $64 \mathrm{~K}$ parts.

Law of the wall profiles shown in Figure 7 with dashed line results in the coefficients of $B=6.7$ and $\kappa=0.43$ observed in the single-phase RE01 simulations and $B=6.7$ and $\kappa=0.42$ for single-phase RE02 cases:

$$
U^{+}=\frac{1}{\kappa} \log y^{+}+B
$$

These are expected constants for the turbulent law of the wall. We have previously observed the values of $B=5.5$ and $\kappa=0.40$ for a rectangular channel which were validated against available data and analytical correlations (Mishra and Bolotnov, 2015). Fluctuations in law of the wall measured above are observed for large $\mathrm{y}^{+}$(200 400 for RE01 and 900 1300 range for RE02) which does not follow classic flat channel behavior. This behavior is related to the geometry of the subchannel: turbulent flow behavior at the center of subchannel is affected by all four rod walls and thus different from the law of the wall in the boundary layer/rectangular geometry. 

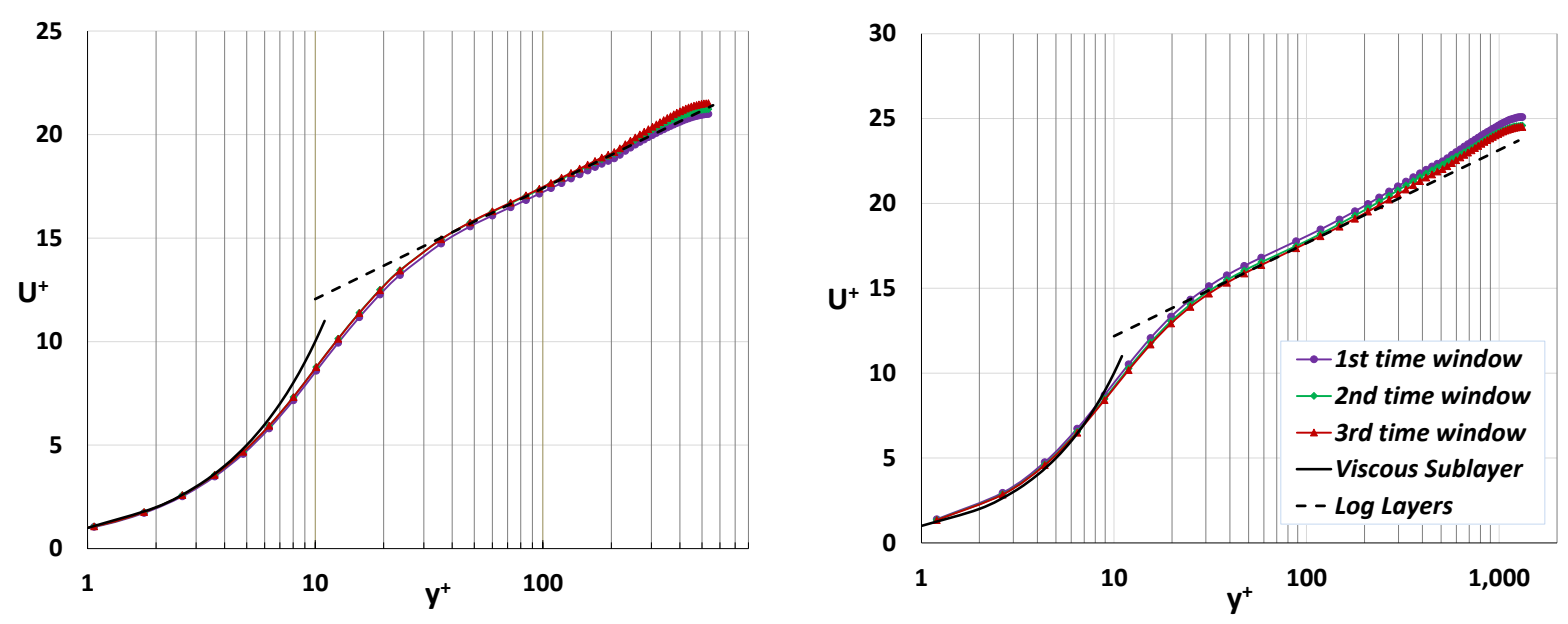

Figure 7. Law of the wall profile for single phase simulations (left: RE01, right: RE02)

The turbulent kinetic energy profile and dimensionless velocity profile are also captured by analyzing the DNS data statistically (Figure 8). Interestingly, there is a flattened region on turbulent kinetic energy's decaying tail for both RE01 and RE02 cases as shown in Figure 8, and distance to the subchannel rod for this inflection is $1.85 \mathrm{~mm}$, which is very close to the half minimum distance between fuel rods $(1.71 \mathrm{~mm}$ in our cases). As we can see in Figure 5, the probes at the same distance to fuel rod wall can experience different turbulent flow near the center of the subchannel compared to the boundaries. The statistical analysis tools we use are averaging the data from the probes located at a constant distance from the walls to produce each of the point in Figure 8. At the larger distance from the wall, beyond the minimum halfdistance between the fuel rods, the averaging occurs over smaller azimuthal region around each fuel rod. This causes the described behavior at the $\mathrm{y}^{+}=250-300$ range shown in Figure 7 for cases RE01 and $\mathrm{y}^{+}=$ 550-600 in case RE02.
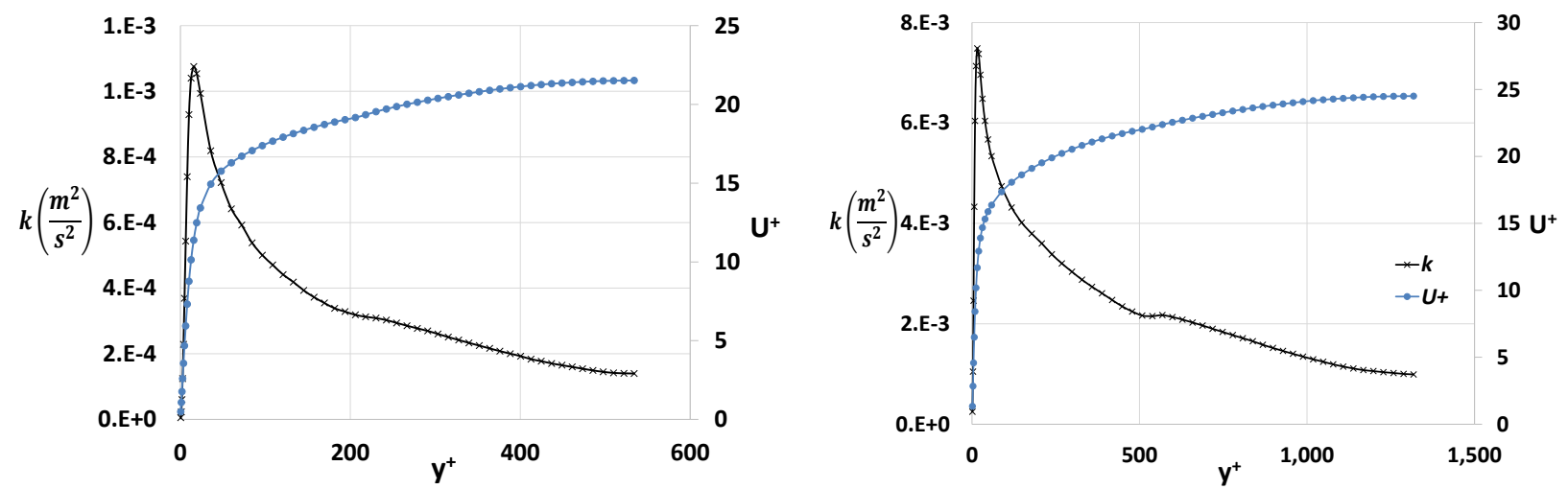

Figure 8. Turbulent kinetic energy and dimensionless velocity for single-phase simulations (left: RE01, right: RE02) 
Once statistically convergent flow is obtained for the single phase subchannel the bubbles are introduced in the domain through the level set method. Generally, two-phase simulations impose stricter requirements on the flow solver, such as smaller CFL number around the bubbles to properly resolve bubble deformation and advection and larger number of iterations at each time step. In addition, more simulation time is needed to accurately compute the bubble void fraction distribution for low void fraction flows due to much smaller data available for the gas phase compared to the liquid phase. The initial condition for the bubbles was specified as the distance field scalar. Seventeen bubbles were randomly initialized in case RE01 and 262 bubbles in case RE02 to represent $1 \%$ gas volume fraction two-phase flow (as shown in Figure 3 and Figure 4). The intent is to obtain statistically significant data in both cases to analyze the void fraction distribution, as well as gas and liquid mean velocity profiles.

The bubble coalescence is a natural phenomenon in bubbly flow due to random collisions among bubbles. However, the standard formulation of level-set interface tracking method has difficulties in accurately representing the bubble coalescence process. Physically, when two bubbles approach, a thin liquid film develops between the bubbles. If this film has sufficient time to drain, then the bubbles will coalesce. Otherwise, the bubbles will bounce off one another. However, the standard level set method will cause coalescence of any bubbles that approach close to one another. Also, since the level-set method uses a smoothed Heaviside function to transition between phase properties, this causes the coalescence process to begin sooner than experimentally observed since the thin liquid film represented by the method has somewhat mixed gas/liquid properties. In order to simulate the coalescence process more accurately, the coalescence control algorithm was developed to prevent or slow the coalescence process. This algorithm locally changes the surface tension on a portion of the bubble surface when it detects that two bubbles approach each other. This local change in surface tension creates a net force that repels the bubbles (Talley, 2014). The coalescence control algorithm has been recently upgraded by being incorporated with advanced bubble tracking capability. In presented bubbly flow simulations (both 17 bubble and 262 bubble cases), this improved coalescence control is employed to better represent the physical behavior of turbulent bubbly flow in PWR subchannel geometry.

As illustrated in Figure 9, Figure 10 and Figure 11, we have statistically processed the recorded data from the two-phase RE01 and RE02 simulations. The turbulent bubbly flows have achieved 8.0 flow-throughs in RE01 case and 1.23 flow-throughs in RE02 cases. Law of the wall analysis shown in Figure 9 with dashed line results in the coefficients of $B=7.8$ and $\kappa=0.5$ observed in the two-phase RE01 simulations and $B=4.8$ and $\kappa=0.35$ for two-phase RE02 cases. Table III summaries the coefficients involved in our law of the wall study. Compared with the results from RE02 simulations, RE01 two-phase cases exhibited 
more fluctuations and the law of the wall profile in has been flattened. One should keep in mind that RE02 two-phase cases can resolve many more bubbles, so larger number of bubble instances will be detected by probes during one flow through. Thus, better statistics were obtained for 262 bubble simulations (RE02) vs. 17 bubble simulation (RE01).

Table III. Summary of coefficients in law of the wall study

\begin{tabular}{|l|r|r|r|}
\hline Case & RE01(Subchannel) & RE02 (Subchannel) & Flat channel \\
\hline Reynolds number $\left(\mathrm{Re}_{h}\right)$ & 29,079 & 80,774 & 29,530 \\
\hline к (single-phase) & 0.43 & 0.42 & 0.40 \\
\hline B (single-phase) & 6.7 & 6.7 & 5.5 \\
\hline к (two-phase) & 0.5 & 0.35 & N/A \\
\hline B (two-phase) & 7.8 & 4.8 & N/A \\
\hline
\end{tabular}
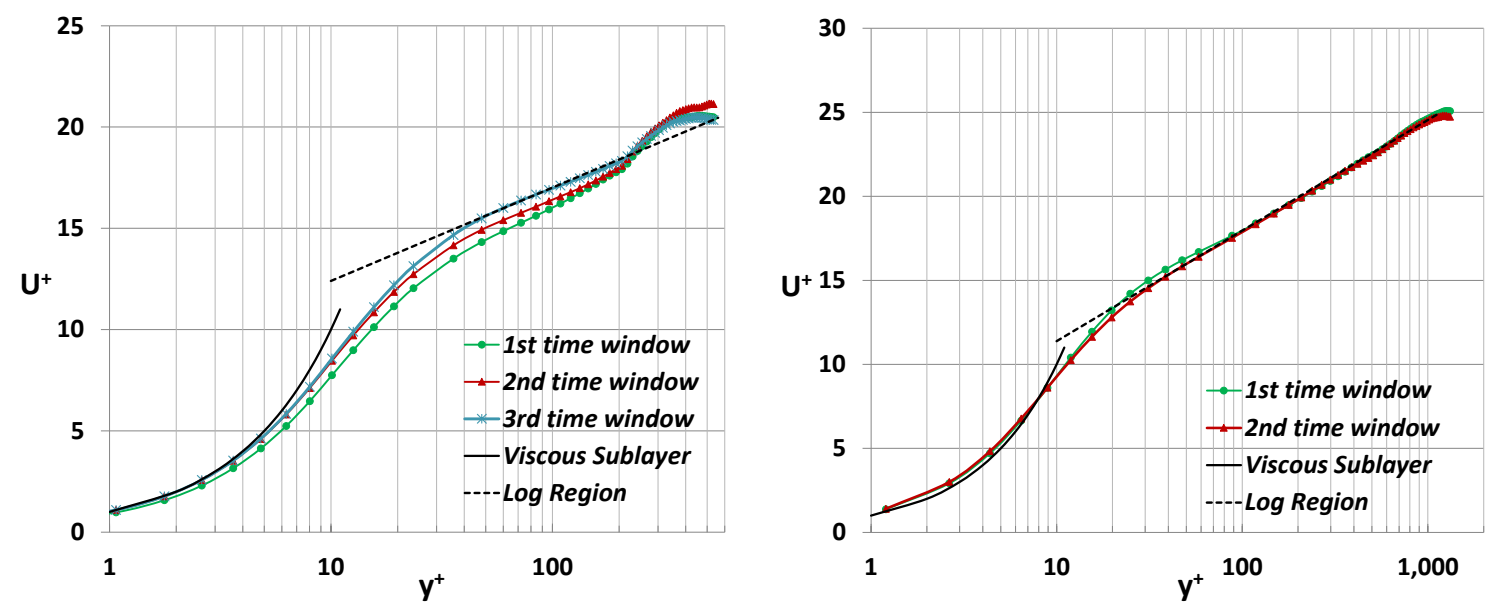

Figure 9. Law of the wall profile for two-phase simulations (left: RE01, right: RE02). The viscous sublayer curve shown is described by $u^{+}=y^{+}$and the log law is described by Eq. (8).

The TKE distributions of both single- and two-phase simulations are plotted and compared in Figure 10. As expected, the bubbles introduce additional energy to turbulence field. The magnitude of TKE in RE02 is notably higher than that in RE01 due to higher Reynolds number in the modeled flow. The distributions of gas and liquid velocity as well as the void fraction from the two-phase RE01 and RE02 simulations are shown in Figure 11. In the region with non-zero void fraction the gas velocity is observed to be larger than liquid velocity because the bubbles are accelerated by the buoyancy force in the subchannel. In both 17 bubble case and 262 bubble case, the void fraction peak shifts closer to the subchannel walls, which is expected because smaller bubbles will migrate in transverse directions due to the effect of the lift force. 
This bubble aggregation behavior towards fuel rod surface is more prominent in case RE02 in which a larger number of smaller bubbles present in the turbulent flow and better statistical data is available.
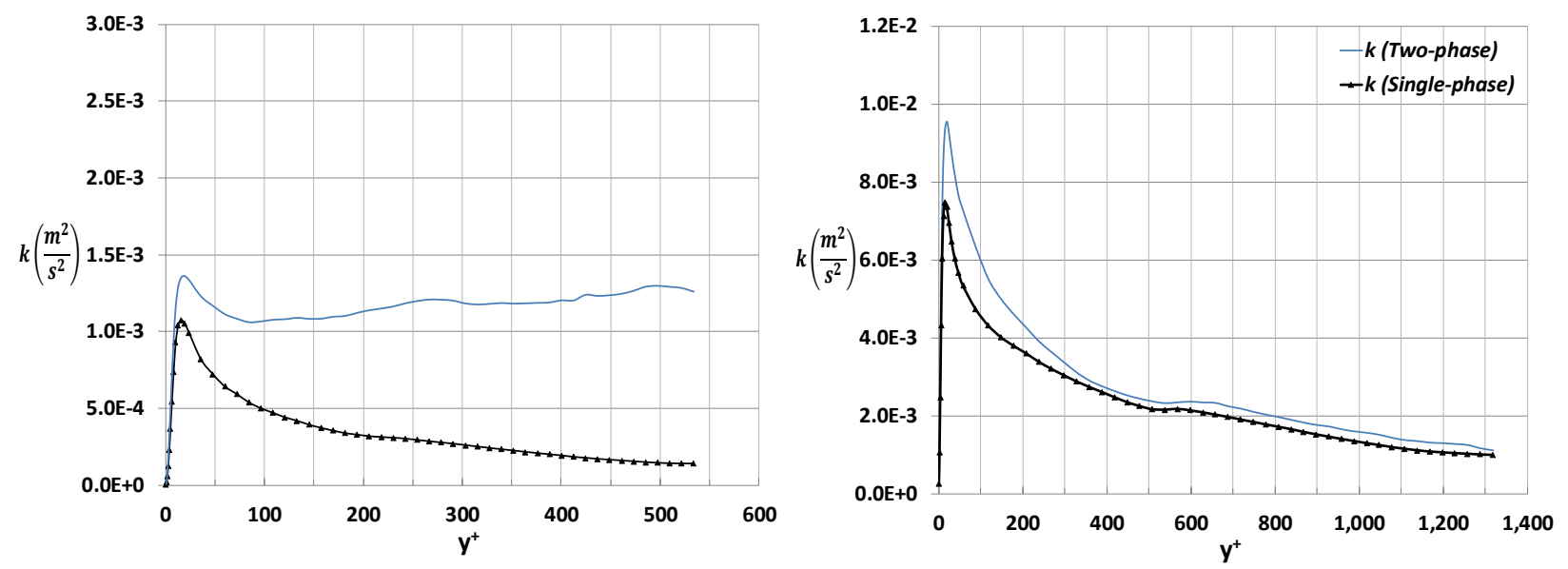

Figure 10. Turbulent kinetic energy profiles of the last time window in single- and two-phase cases (left: RE01, right: RE02).
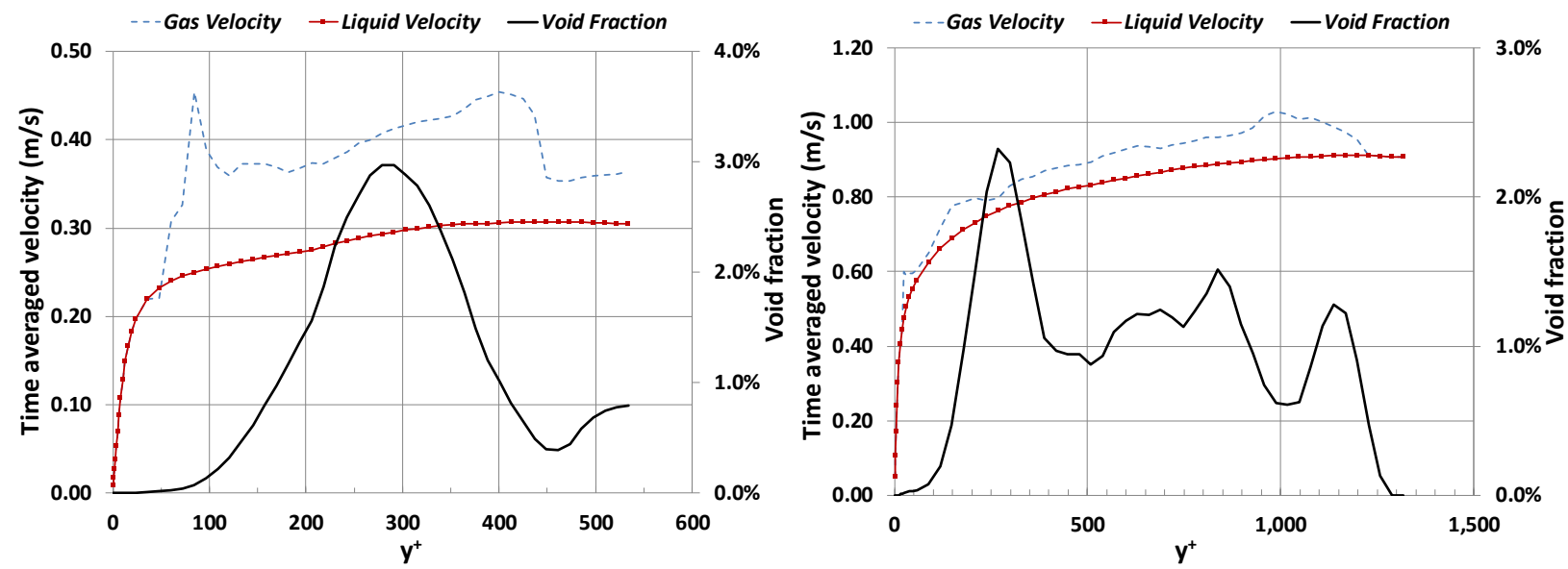

Figure 11. Void fraction and gas-liquid velocity profiles of the last time window in two-phase simulations (left: RE01, right: RE02).

In the two-phase flow simulations performed herein, bubbles are introduced when single-phase turbulence has reached the steady state conditions. The further development of turbulence field is primarily attributed to the interaction between bubbles and liquid turbulence. In experiments the channel hydraulic diameter is commonly used to quantify the length scale needed for fully developed single-phase turbulence. As an analogy, we propose to use average bubble diameter to quantify the length scale needed for a fully fulfillment of bubbles' impact on steady-state single-phase turbulence field. From this perspective, smaller bubbles will result in more bubble diameter units compared to larger bubbles in the same geometry. As for our simulations in single PWR subchannel geometry, a single flow-through corresponds 
to about 62 bubble diameter units for RE02 262-bubble case. In RE02 case the bubbles have moved 76 bubble diameter length, which should be enough for two-phase flow turbulence to further develop considering the prior single-phase steady-state turbulence. When two-phase flows achieve statistically steady state conditions, the drag coefficient can be estimated based on the bubbly buoyancy force and bubble terminal velocity. Assuming that the steady state conditions are reached, the bubble buoyancy force will balance out the drag force. By averaging the bubble relative velocities, the following equation can be used to estimate the drag coefficient $\left(C_{d}\right)$ in RE02 case:

$$
F_{d}=\frac{1}{2} \rho_{l} v_{r}^{2} C_{d} A_{b u b b l e}=\left(\rho_{l}-\rho_{g}\right) g V_{\text {bubbble }}
$$

where $\rho_{l}$ and $\rho_{g}$ are liquid and gas densities; $v_{r}$ is averaged bubble relative velocity in streamwise direction; $A_{\text {bubble }}$ and $V_{\text {bubbble }}$ are averaged cross-sectional area and volume of a bubble. We chose not to estimate the drag coefficient in the 17 bubble case due to unrealistically large bubbles. The resultant

drag coefficient is 0.071 in RE02 two-phase case. According to Tomiyama drag correlation for single bubbles in standing liquid (Tomiyama et al., 1998), the suggested drag coefficient is 0.1617 for case RE02. As mentioned earlier, our approach was used to re-produce the drag coefficient correlation by Tomiyama with less than $1 \%$ error for single spherical bubbles in the past (Thomas et al., 2015). Using the same numerical tools, we have shown that the drag coefficient is about half of the single bubble in standing fluid correlation in realistic flows in the reactor subchannel. Multiple bubble interaction results in bubble clustering which allows higher relative velocities effectively reducing the drag force experiencing by any single bubble. Liquid turbulence also has an effect on bubble motion compared to the standing fluid assumption.

\section{Conclusion}

Latest results on DNS/ITM simulations in subchannel flow geometry for single and two-phase turbulent flows are presented. While the computational cost remains a big challenge for larger domains and high Reynolds number simulations, the current capabilities clearly demonstrate that DNS/ITM approach can be a valuable and promising tool to guide the development of CMFD models as well as subchannel closure laws for nuclear reactor applications. We have shown the single-phase law of the wall constants from the subchannel turbulence are similar to the ones observed in the parallel plates geometry, and the constants may change in the presence of bubbles. The bubble aggregation near the fuel rods is observed in the 262 
bubble RE02 case as expected due to the lift force effect. Although the static virtual probe approach has successfully captured some interesting two-phase flow behavior, it lacks the ability to collect detailed parameters regarding individual bubble behavior. A bubble tracking methodology has been developed which will bring in-depth insights of two-phase turbulent flows. Future work will include performing more representative simulations for nuclear reactor designs, as well as the development of advanced analysis techniques that can statistically analyze individual bubble behavior. These studies will help to assess the state-of-the-art multiphase computational fluid dynamics (M-CFD) models, and find out more accurate closure laws to improve the prediction accuracy of turbulent flows of nuclear engineering interests.

\section{Acknowledgement}

The authors would like to acknowledge the support from Consortium for Advanced Simulation of Light Water Reactors (http://www.casl.gov), an Energy Innovation Hub (http://www.energy.gov/hubs) for Modeling and Simulation of Nuclear Reactors under U.S. Department of Energy [grant number DEAC05-00OR22725]. An award of computer time was provided by the ASCR Leadership Computing Challenge (ALCC) program. This research used resources of the Argonne Leadership Computing Facility, which is a DOE Office of Science User Facility supported under Contract DE-AC02-06CH11357. The solution presented herein made use of the Acusim linear algebra solution library provided by Altair Engineering Inc. and meshing and geometric modeling libraries by Simmetrix Inc.

\section{Reference}

Anglart, H., Nylund, O., 1996. CFD application to prediction of void distribution in two-phase bubbly flows in rod bundles. Nucl. Eng. Des. 163, 81-98.

Avramova, M., 2007. Development of an innovative spacer grid model utilizing computational fluid dynamics within a subchannel analysis tool.

Baglietto, E., Ninokata, H., Misawa, T., 2006. CFD and DNS methodologies development for fuel bundle simulations. Nucl. Eng. Des. 236, 1503-1510.

Bolotnov, I.A., 2013. Influence of Bubbles on the Turbulence Anisotropy. Journal of Fluids Engineering $135,051301$. 
Bolotnov, I.A., Jansen, K.E., Drew, D.A., Oberai, A.A., Lahey, J.T.,R., 2011. Detached Direct Numerical Simulation of Turbulent Two-phase Bubbly Channel Flow. Int. J. Multiphase Flow 37, 647-659.

Brackbill, J.U., Kothe, D.B., Zemach, C., 1992. A continuum method for modeling surface tension. Journal of computational physics 100, 335-354.

Carajilescov, P., Todreas, N., 1976. Experimental and analytical study of axial turbulent flows in an interior subchannel of a bare rod bundle. Journal of Heat Transfer 98, 262-268.

Conner, M.E., Baglietto, E., Elmahdi, A.M., 2010. CFD methodology and validation for single-phase flow in PWR fuel assemblies. Nucl. Eng. Des. 240, 2088-2095.

Dabiri, S., Lu, J., Tryggvason, G., 2013. Transition between regimes of a vertical channel bubbly upflow due to bubble deformability. Physics of Fluids (1994-present) 25, 102110.

Dominguez-Ontiveros, E.E., Hassan, Y.A., 2009. Non-intrusive experimental investigation of flow behavior inside a $5 \times 5$ rod bundle with spacer grids using PIV and MIR. Nucl. Eng. Des. 239, 888-898.

Fang, J., Mishra, A.V., Bolotnov, I.A., 2014. Interface Tracking Simulation of Two-phase Bubbly Flow in A PWR Subchannel.

Fang, J., Thomas, A.M., Bolotnov, I.A., 2013. Development of Advanced Analysis Tools for Interface Tracking Simulations. 109, 1613-1615.

Fatemi, E., Sussman, M., 1995. An efficient interface preserving Level-Set Re-distancing algorithm and its application to interfacial incompressible fluid flow. SIAM J Sci Statist Comput 158, 36.

Jansen, K.E., 1993. Unstructured grid large eddy simulations of wall bounded flows. Annual Research Briefs, Center for Turbulence Research, NASA Ames/Stanford University, 151.

Jansen, K.E., 1999. A stabilized finite element method for computing turbulence. Comput. Methods Appl. Mech. Eng. 174, 299-317.

Kim, J., Moin, P., Moser, R., 1987. Turbulence statistics in fully developed channel flow at low Reynolds number. J. Fluid Mech. 177, 133-166.

Končar, B., Kljenak, I., Mavko, B., 2004. Modelling of local two-phase flow parameters in upward subcooled flow boiling at low pressure. Int. J. Heat Mass Transfer 47, 1499-1513.

Lee, M., Malaya, N., Moser, R.D., 2013. Petascale direct numerical simulation of turbulent channel flow on up to $786 \mathrm{k}$ cores. , 61 .

Lu, J., Tryggvason, G., 2008. Effect of bubble deformability in turbulent bubbly upflow in a vertical channel. Physics of Fluids (1994-present) 20, 040701. 
Mishra, A.V., Bolotnov, I.A., 2015. DNS of turbulent flow with hemispherical wall roughness. Journal of Turbulence 16, 225-249.

Moser, R.D., Kim, J., Mansour, N.N., 1999. Direct numerical simulation of turbulent channel flow up to $\operatorname{Re}=590$. Phys. Fluids 11, 943-945.

Nagrath, S., Jansen, K., Lahey Jr, R.T., Akhatov, I., 2006. Hydrodynamic simulation of air bubble implosion using a level set approach. Journal of Computational Physics 215, 98-132.

Ninokata, H., Atake, N., Baglietto, E., Misawa, T., Kano, T., 2004. Direct numerical simulation of turbulent flows in a subchannel of tight lattice fuel pin bundles of nuclear reactors. Annual Report of the Earth Simulator Center April 2005.

Orszag, S.A., 1970. Analytical theories of turbulence. J. Fluid Mech. 41, 363-386.

Pope, S.B., 2000. Turbulent Flows. Cambridge university press.

Rasquin, M., Smith, C., Chitale, K., Seol, E.S., Matthews, B.A., Martin, J.L., Sahni, O., Loy, R.M., Shephard, M.S., Jansen, K.E., 2014. Scalable Implicit Flow Solver for Realistic Wing Simulations with Flow Control. Computing in Science \& Engineering 16, 13-21.

Rehme, K., 1989. Experimental observations of turbulent flow through subchannels of rod bundles. Exp. Therm. Fluid Sci. 2, 341-349.

Sahni, O., Müller, J., Jansen, K., Shephard, M., Taylor, C., 2006. Efficient anisotropic adaptive discretization of the cardiovascular system. Comput. Methods Appl. Mech. Eng. 195, 5634-5655.

Sethian, A.J., 1999. Level Set Methods and Fast Marching Methods. Cambridge University Press.

Spalart, P.R., Deck, S., Shur, M.L., Squires, K.D., Strelets, M.K., Travin, A., 2006. A New Version of Detached-eddy Simulation, Resistant to Ambiguous Grid Densities. Theor. Comput. Fluid Dyn. 20, 181195.

Sussman, M., Almgren, A.S., Bell, J.B., Colella, P., Howell, L.H., Welcome, M.L., 1999. An adaptive level set approach for incompressible two-phase flows. Journal of Computational Physics 148, 81-124.

Sussman, M., Fatemi, E., Smereka, P., Osher, S., 1998. An improved level set method for incompressible two-phase flows. Comput. Fluids 27, 663-680.

Talley, M.L., 2014. Bubble coalescence control development for level set interface tracking method.

Tejada-Martínez, A.E., Jansen, K.E., 2006. A parameter-free dynamic subgrid-scale model for large-eddy simulation. Comput. Methods Appl. Mech. Eng. 195, 2919-2938.

Thomas, A.M., Fang, J., Feng, J., Bolotnov, I.A., 2015. Estimation of shear-induced lift force in laminar and turbulent flows. Nuclear Technology 109, 1-18. 
Thurgood, M.J., 1983. COBRA/TRAC, a Thermal-Hydraulics Code for Transient Analysis of Nuclear Reactor Vessels and Primary Coolant Systems. The Commission.

Tomiyama, A., Kataoka, I., Zun, I., Sakaguchi, T., 1998. Drag coefficients of single bubbles under normal and micro gravity conditions. JSME international journal.Series B, fluids and thermal engineering 41, 472-479.

Trofimova, A.V., Tejada-Martínez, A.E., Jansen, K.E., Lahey Jr, R.T., 2009. Direct numerical simulation of turbulent channel flows using a stabilized finite element method. Comput. Fluids 38, 924-938.

Trupp, A.C., Azad, R., 1975. The structure of turbulent flow in triangular array rod bundles. Nucl. Eng. Des. 32, 47-84.

Ustinenko, V., Samigulin, M., Ioilev, A., Lo, S., Tentner, A., Lychagin, A., Razin, A., Girin, V., Vanyukov, Y., 2008. Validation of CFD-BWR, a new two-phase computational fluid dynamics model for boiling water reactor analysis. Nucl. Eng. Des. 238, 660-670.

Whiting, C.H., Jansen, K.E., 2001. A stabilized finite element method for the incompressible NavierStokes equations using a hierarchical basis. Int. J. Numer. Methods Fluids 35, 93-116.

Winters, J., Vijuk, R., Cummins, W., 2004. AP1000 Design Control Document. Weistinghouse Electric Company LLC, Pittsbrugh, PA, US .

Wu, X., Trupp, A., 1993. Experimental study on the unusual turbulence intensity distributions in rod-towall gap regions. Exp. Therm. Fluid Sci. 6, 360-370. 